ISAHP 1999, Kobe, Japan, August 12-14, 1999

\title{
AN EVALUATION OF PASSENGERS' ALTERNATIVE INTERURBAN TRANSPORTATION
}

\author{
Simin Jalali R. Rabbani, D. Sc. \& Soheil Rahnemay Rabbani, D.Sc. \\ Civil Engineering Department, Center of Science and Technology \\ Federal University of Paraiba-UFPb, \\ Rua Marechal Deodoro, 631 Bairro: Prata 58108-530 Campina Grande- PB, Brasil \\ Fone: (083) 342 0908; Fax: (083) 3420945 simin@dsc.ufpb.br
}

Public transportation in Brazil is used mainly for interurban movements. More recently, however, public transportation deficiencies such as insufficient fleet, crowded buses and lack of safety, accompanied by increasing unemployment rate, have given birth to an alternative mode of transportation. This kind of transportation, whether by moto-taxi, minibus, or car/van-pooling, has been the subject of extensive discussion by different groups of politicians, authorities, and public representatives, at federal, state, and local levels. Some support the idea of regulation, regularization and institutionalization of altemative transportation because it provides personal security, an organized timetable, and relatively adequate public attendance; and especially for providing during current social crisis. Others definitely oppose this kind of transportation for its lack of safety and lack of contributions to government revenue through taxes. The users have their own divergent opinions, but all prefer the existence of this alternative mode of transportation, accompanied with some kind of regulation of the system.

Although this mode of transportation has been operating in Brazil for more than 20 years, it is only now spreading throughout the country; reflecting the population's need for faster, safer, more flexible transportation than the existing public system provides. It is believed that the growth of this alternative mode of transportation indicates the need to restructure the public transportation service, especially in urban areas.

Since our bibliographical research indicates that no study has been conducted to evaluate this kind of alternative system, we intend to present here a methodology for a multicriterial evaluation of the interurban passenger transportation system, which will incorporate relevant objectives and interests of the users, public authorities, operators of the alternative system, operators of the interurban bus service, and the society as a whole.

The Analytic Network Process (ANP), developed by Thomas L. Saaty, constitutes the basis of the methodology proposed here. It takes into account principal interest groups involved in or affected by the decision making process, as well as their relevant objectives and interests, whether easily quantifiable or not. The systematic nature of the method allows a more refined examination of costs and benefits from social, economic, political and environmental points of view.

In order to verify the applicability of the methodology, it was applied to the intercity transportation of passengers in the State of Paraiba, particularly in Campina Grande, a medium-sized city that is considered one of the principal centers of the economy of the state, as well as the second major city in northeast Brazil.

The data necessary for the application of the methodology was collected through questionnaires, visits to principal entities, and local observations. The data was classified into three groups: data related to the users of the system, data related to the operators of the alternative system, and data related to the regular bus service operators of the intermunicipal passenger transportation system. The systematic analysis of the problem required the judgement of many specialists on economic, social, political, and environmental aspects. Different specialists were informed of the nature of the problem and were asked to make necessary judgments on this application. 
An analysis of the results of the application of the methodology indicate the relative priority of alternative plans of action to be as follows (listed in order of importance): the Implementation of Managerial Programs (relative importance of 0.36), the Implementation of Mixed Fleet with different size vehicles (0.24), Legalization and Regulation of Alternative Modes of Transportation (0.17), the Null Alternative of Remaining in the Same Situation (0.13) and, finally, the Prohibition of Informal Activity (0.10).

Although the results of the application of the methodology indicate that the implementation of managerial programs is the most important factor in improving the level of service in order to recover those passengers who abandoned the interurban public bus service, it is worth mentioning that it is not intended to indicate the most important alternative, but only to establish relative priorities for the alternative plans of action. 